\title{
High Energy Nuclear Collisions: Constituent Participant Scaling and Suppression in Forward Production
}

\section{Bożena BOIMSKA*i}

The Andrzej Sołtan Institute for Nuclear Studies, Hoża 69, PL-00-681, Warsaw, POLAND

E-mail: boimska@fuw.edu.pl

\begin{abstract}
A good understanding of different effects in particle production from nuclear collisions at LHC will require, first, understanding of the data at lower energies - in particular, these measured at SPS and RHIC energies. In this work, results on particle multiplicities and transverse momentum spectra are reported. It is known from the past studies, that particle multiplicities from high energy nuclear collisions can be parametrized and/or explained within participant ("wounded") nucleon or quark frameworks. Examples illustrating successes and failures of these two approaches are discussed. The latest results on identified particle production from the NA49 experiment at the CERN-SPS accelerator are shown. The study was done for both the nucleon and quark participant frameworks using the nuclear overlap model. In addition, results concerning transverse momentum spectra, expressed in terms of a so-called nuclear modification factor, of particles from hadron-nucleus collisions are presented. In the forward region at RHIC, particle production is suppressed - the effect often interpreted as a manifestation of saturation and/or Color Glass Condensate. Preliminary results for the SPS energy show a similar suppression, as presented and discussed in this work.
\end{abstract}

XXth Hadron Collider Physics Symposium

November 16 - 20, 2009

Evian, France

\footnotetext{
${ }^{*}$ Speaker.

${ }^{\dagger}$ B. B. would like to thank the organizers of HCP2009 for the financial support and their hospitality during the conference.
} 


\section{Constituent Participant Scaling}

Particle multiplicities measured for hadron-hadron $(h+h)$, hadron-nucleus $(h+A)$ and nucleusnucleus $(\mathrm{A}+\mathrm{A})$ reactions provide information about the system formed in these collisions. In the past, many attempts to describe (parametrize) the amount of produced particles were undertaken. For example, within the Wounded Nucleon Model (WNM)[1] particle production in a nuclear collision was predicted to be a superposition of independent contributions from the wounded $^{1}$ nucleons (called also participant nucleons ) in the projectile and the target. For $\mathrm{h}+\mathrm{A}$ collisions, for the total charged multiplicity, WNM works very well, up to the highest RHIC energy [2]. However, it fails for nucleus-nucleus reactions [3]. Still, proportionality of the multiplicity in A+A to the number of participant nucleons holds.

What happens for identified particle production? The study was done for pion and kaon multiplicities, based on the NA49 data, from $\mathrm{C}+\mathrm{C}, \mathrm{Si}+\mathrm{Si}$ [4] and $\mathrm{Pb}+\mathrm{Pb}$ [5] collisions at the top SPS energy. The nuclear overlap model [6] was employed to calculate the number of participating objects - nucleons $\left(N_{n-\text { part }}\right)$ and constituent-quarks $\left(N_{q-p a r t}\right)$. The thus obtained numbers of participant nucleons and quarks were used to normalize the particle yields measured by NA49. The normalized yields are shown in Fig. 1. For A+A collisions at SPS, the total pion yield was found to scale with $N_{n-p a r t}$ and kaon yield with $N_{q-\text { part }}$.
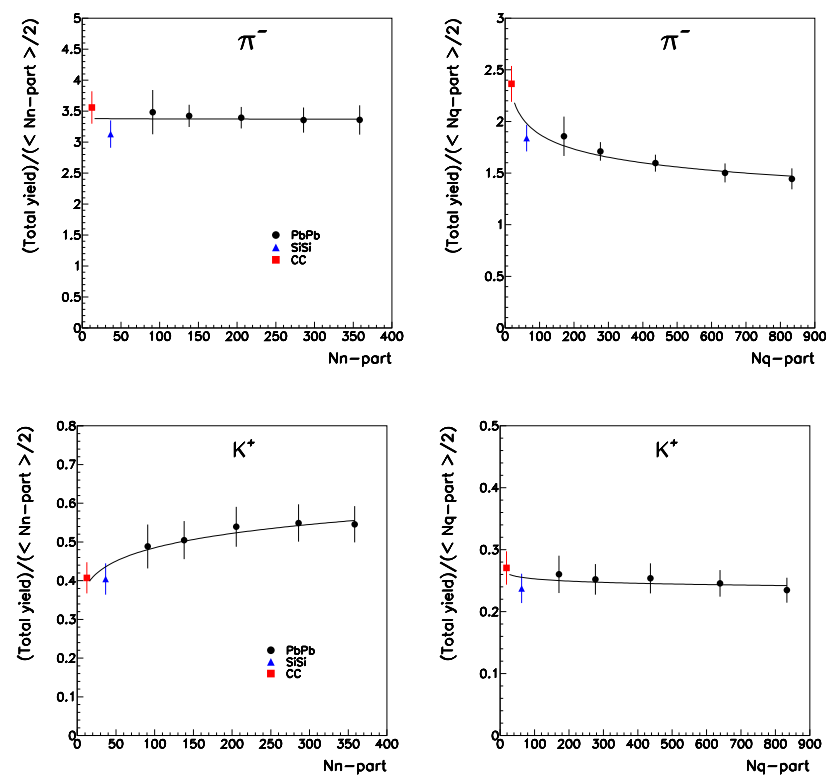

Figure 1: Total yields of $\pi^{-}$and $\mathrm{K}^{+}$, for $\mathrm{C}+\mathrm{C}, \mathrm{Si}+\mathrm{Si}$ and $\mathrm{Pb}+\mathrm{Pb}$ collisions at $\sqrt{s_{N N}}=17.2 \mathrm{GeV}$, scaled by participant nucleons and quarks (left and right panels, respectively).

\section{Suppression in Forward Production}

Transverse momentum $\left(p_{T}\right)$ spectra of particles from $\mathrm{A}+\mathrm{A}$ collisions are subject to initial and final state effects. In $\mathrm{h}+\mathrm{A}$ interactions only the initial state ones are present. The behavior of $p_{T}$ spectra is studied in terms of the Nuclear Modification Factor (NMF), which for $\mathrm{h}+\mathrm{A}$ is defined as:

$$
R_{h A}\left(p_{T}, y\right)=\frac{1}{\left\langle N_{\text {coll }}\right\rangle} \frac{d^{2} N^{h A} / d p_{T} d y}{d^{2} N^{p p} / d p_{T} d y},
$$

where: $N_{\text {coll }}$ - is the number of binary collisions. Experiments at RHIC studied the evolution of NMF with (pseudo)rapidity. Particle production in $\mathrm{d}+\mathrm{Au}$ collisions at $\sqrt{s_{N N}}=200 \mathrm{GeV}$ is sup-

\footnotetext{
${ }^{1} \mathrm{~A}$ wounded nucleon is a nucleon that underwent at least one inelastic collision.
} 

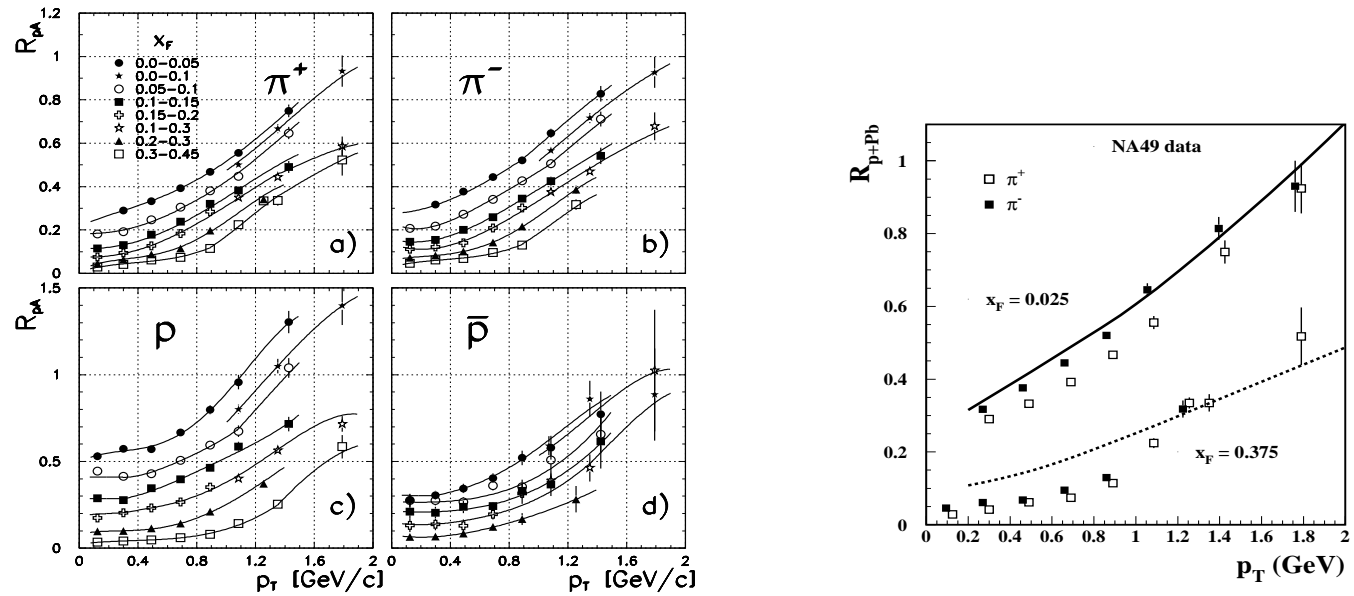

Figure 2: Left: Evolution of NMF with $x_{F}$ for a) $\pi^{+}$, b) $\pi^{-}$, c) $p$ and d) $\bar{p}$ from central $\mathrm{p}+\mathrm{Pb}$ collisions at $\sqrt{s_{N N}}=17.2 \mathrm{GeV}$. Right: Model calculations and experimental data on NMF for pions, from $\mathrm{p}+\mathrm{Pb}$ collisions at the SPS energy, at $x_{F}=0.025$ and 0.375 .

pressed in the forward region - NMF decreases when (pseudo)rapidity increases [7, 8]. The suppression can be qualitatively described within the Color Glass Condensate (CGC) model [9], which assumes gluon saturation for the kinematic domain reached in hadron-nucleus collisions at RHIC. What happens at lower energies? The analysis, done by NA49, of identified hadrons produced in $\mathrm{p}+\mathrm{Pb}$ collisions showed decreasing values of NMF with increasing $x_{F}$ (left part of Fig. 2) [10]. Although the effects related to CGC are not expected to be present at the SPS energy, the measured suppression is similar to that at RHIC. Theoretical studies of the energy dependence, based on the Glauber-Gribov model in which the energy-momentum conservation was implemented into the multiple soft parton rescattering approach (CGC not included), predict the suppression both at RHIC and SPS energies. The description of the NA49 data is, however, not very good (right panel of Fig. 2) [11]. This shows, that understanding of the suppression and its energy dependence needs more theoretical investigation.

\section{References}

[1] A. Białas, M. Bleszyński, W. Czyż, Nucl. Phys. B 111 (1976) 461.

[2] B. B. Back et al. (PHOBOS Collaboration), Nucl. Phys. A 757 (2005) 28.

[3] B. Alver et al., Phys. Rev. Lett. 102 (2009) 142301.

[4] C. Alt et al. (NA49 Collaboration), Phys. Rev. Lett. 94 (2005) 052301.

[5] P. Dinkelaker, Ph.D. Thesis, University of Frankfurt (2009); results to be published soon.

[6] K. J. Eskola, K. Kajantie, J. Lindfors, Nucl. Phys. B 323 (1989) 37.

[7] I. Arsene et al. (BRAHMS Collaboration), Phys. Rev. Lett. 93 (2004) 242303.

[8] J. Adams et al. (STAR Collaboration), Phys. Rev. Lett. 97 (2006) 152302.

[9] D. Kharzeev et al., Phys. Rev. D 68 (2003) 094013.

[10] B. Boimska, Ph.D. Thesis, Institute for Nuclear Studies, Warsaw (2004), CERN-THESIS-2004-035.

[11] J. Nemchik and M. Šumbera, arXiv:0907.4062; to be published in Nucl. Phys. A 